\title{
The emergence of the two cell fates and their associated switching for a negative auto-regulating gene
}

\author{
Zhenlong Jiang ${ }^{1,3+}, \mathrm{Li}_{\operatorname{Tian}^{1 \dagger}}$, Xiaona Fang ${ }^{1 \dagger}$, Kun Zhang $^{1 \dagger}$, Qiong Liu ${ }^{1 \dagger}$, Qingzhe Dong ${ }^{1}$, Erkang Wang ${ }^{1}$ and \\ Jin Wang 2* $^{*}$
}

\begin{abstract}
Background: Decisions in the cell that lead to its ultimate fate are important for fundamental cellular functions such as proliferation, growth, differentiation, development, and death. These cell fate decisions can be influenced by both the gene regulatory network and also environmental factors and can be modeled using simple gene feedback circuits. Negative auto-regulation is a common feedback motif in the gene circuits. It can act to reduce gene expression noise or induce oscillatory expression and is thought to lead to only one cell fate. Here, we present experimental and modeling data to suggest that a self-repressor circuit can lead to two cell fates under specific conditions.
\end{abstract}

Results: We show that the introduction of inducers capable of binding and unbinding to a self-repressing gene product (protein), thus regulating the associated gene, can lead to the emergence of two cell states. We suggest that the inducers can alter the effective regulatory binding and unbinding speed of the self-repressor regulatory protein to its destination DNA without changing the gene itself. The corresponding simulation results are consistent with the experimental findings. We propose physical and quantitative explanations for the origin of the two phenotypic cell fates.

Conclusions: Our results suggest a mechanism for the emergence of multiple cell fates. This may explain the heterogeneity often observed among cell states, while illustrating that altering gene regulation strength can influence cell fates and their decision-making processes without genetic changes.

Keywords: Gene expression, Self-repressor, Bimodality, Cell fate decision-making

\section{Background}

Uncovering the origin of the phenotypes or fates of the cell and their associated switching is important for the full understanding of cell functions such as proliferation, growth, differentiation, development, and death. This remains a challenging issue in biology. It is clear that the underlying gene regulatory networks are crucial in determining the function of the cell [1-6]. Usually, phenotypes of organisms or cells can be determined and are

\footnotetext{
* Correspondence: jin.wang.1@stonybrook.edu

${ }^{\dagger}$ Zhenlong Jiang, Li Tian, Xiaona Fang, Kun Zhang and Qiong Liu contributed equally to this work.

${ }^{2}$ Department of Chemistry, Physics and Applied Mathematics, State

University of New York at Stony Brook, Stony Brook, New York 11794-3400,

USA

Full list of author information is available at the end of the article
}

measurable. A phenotype is often thought to be determined by the genotype in the conventional perspective [7-11]. Recently, some studies have indicated that microenvironments or epigenetics can also alter the fates of the cell or its phenotypes even with the same genotypes [12-21]. In other words, there is a possibility that apart from mutating the genes or the nodes themselves in the gene circuit, changing the underlying gene regulatory wirings among the genes or nodes in the regulatory network can alter the cell phenotypes or fates. In this study, we aim to study how altering gene regulation determines cell fates.

Negative auto-regulation is abundant: it is found in nearly $50 \%$ of the feedback loops in gene regulatory networks. It is widely believed that negative auto-regulation

(c) The Author(s). 2019 Open Access This article is distributed under the terms of the Creative Commons Attribution 4.0 International License (http://creativecommons.org/licenses/by/4.0/), which permits unrestricted use, distribution, and reproduction in any medium, provided you give appropriate credit to the original author(s) and the source, provide a link to the Creative Commons license, and indicate if changes were made. The Creative Commons Public Domain Dedication waiver (http://creativecommons.org/publicdomain/zero/1.0/) applies to the data made available in this article, unless otherwise stated. 
leads to a reduction of the gene expression noise, an increase of gene response times, an induction of possible oscillatory gene expression, and an improvement of the stability of proteins produced by the underlying gene networks [22-26]. Despite these novel findings, most experimental studies have been focused on the influences of the genetic structures themselves, rather than the environmental or the epigenetic effects on the selfrepressor.

For a self-repressing system, the expression distribution is commonly more concentrated and welldistributed as a unimodal distribution [27]. Many previous investigations have reached similar conclusions, observing only one cell fate $[25,28-30]$. However, these experiments were performed mostly in simple organisms such as bacteria, for which it is often assumed that the speed of regulatory protein binding/unbinding to the corresponding DNA for switching is significantly faster than the synthesis and degradation of the corresponding regulatory proteins. In fact, in most organisms, cell complexes such as the nuclei inside mammalian cells may give rise to effectively slower processes of the underlying gene regulatory binding/unbinding, due to environmental complexities such as epigenetic effects through histone modification or DNA methylation. That is, the effective rates of binding/unbinding of the regulatory proteins to the DNA can be comparable to, or even slower than, the production and degradation rate of the regulatory proteins [31]. Modeling studies [32-34] indicate that, in this case, the protein expressions of a negative feedback loop motif may not always show a simple single steady state, but instead can show two steady states, resulting in two different cell fates. Since the auto-regulation circuit involves only a single gene, it is the simplest gene regulation in vivo. We will show experimentally that this simple gene auto-regulation circuit can lead to different cell fates or phenotypes under specific conditions, rather than that of only one cell fate as is commonly expected.

\section{Results}

\section{Self-repressing gene circuit and non-regulatory gene} circuit

In this study, we have designed and constructed a purely negative auto-regulation feedback loop circuit (selfrepressing gene circuit) in Escherichia coli (E. coli). The Ptet promoter including two TetO operons controls the production of its repressor, TetR. Meanwhile, the TetR was fused with a fluorescence protein (Venus) for experimental measurements of the TetR expressions. The inducer, aTc (anhydrotetracycline), was introduced to regulate expressions of the self-repression system. In the presence of an inducer, the repressor TetR can change its conformation and dissociate from specific binding sequences of the DNA (TetO). This allows for the transcription of TetR-Venus (Fig. 1a). In order to avoid fluctuations in copy numbers of the plasmids, the constructed circuit in the plasmid was integrated into the chromosome of E. coli. We also constructed a series of self-repressing circuits with different affinities to the TetR protein (MG::PR-WT, MG::PR-1G) (Additional file 1: Figure S2). We chose MG::PR-8 $\mathrm{T}$ as the main circuit of this study for its stability and bimodal behavior. To compare this with our self-repressing circuit construction MG::PR-8 T, we designed a non-regulatory circuit as a control group: the MG::PR-8 T-P39K circuit (Fig. 1b).

\section{The expression distributions of the self-repressor gene circuit under microscopy}

To obtain the expressions of TetR under different induction conditions, we measured the average fluorescence signals of the reporter protein Venus for the strain of MG::PR-8 T at different inducer concentrations (300$1500 \mathrm{ng} / \mathrm{mL}$ ) across cell populations using a wide-field fluorescence microscope. Cells were collected and measured after being cultured in M9 medium and induced by aTc for $4 \sim 6 \mathrm{~h}$ to a logarithmic phase. To ensure accuracy of the expression distribution, we collected no less than $10^{3}$ cells to measure for each sample. All expression distributions under different induction concentrations are shown in Fig. 2. The results indicate that $T e t R$ expression distributions vary with inducer (aTc) concentrations. Under low inducer concentrations, the expression levels of the negative regulated gene circuit were quite low, and this gene can be considered to be in the "off" state for a long time. With increased inducer concentrations, the expression levels were significantly enhanced (Fig. 2a). From the results shown in the microscope, we can clearly see that when inducers are added to the system, the repressor TetR can no longer prevent the transcription of TetR. When the inducer concentrations are high enough (such as $1400 \mathrm{ng} / \mathrm{mL}$ and 1500 $\mathrm{ng} / \mathrm{mL}$ ), the steady state expression distribution can become bimodal, with two states of low and high expression levels. Meanwhile, the percentage of the cells in the low expression state gradually increases with the increase of the inducer concentrations (Fig. 2a). Under high inducer concentrations, the coexistence of both phenotypes characterized by the bimodal steady state distributions of the fluorescence intensities can be clearly seen (Fig. 2e). When we further compare the images in Fig. 2d and e, it is clear to see that one section of the cells in Fig. 2e is brighter, while other sections were dimmer, compared to most of the cells in Fig. 2d. As can be seen from the microscopy images, the morphologies of the bacteria cells are not influenced by the aTc inducers at a concentration level of $1500 \mathrm{ng} / \mathrm{mL}$ (Fig. 2e). The corresponding distributions of those images are 


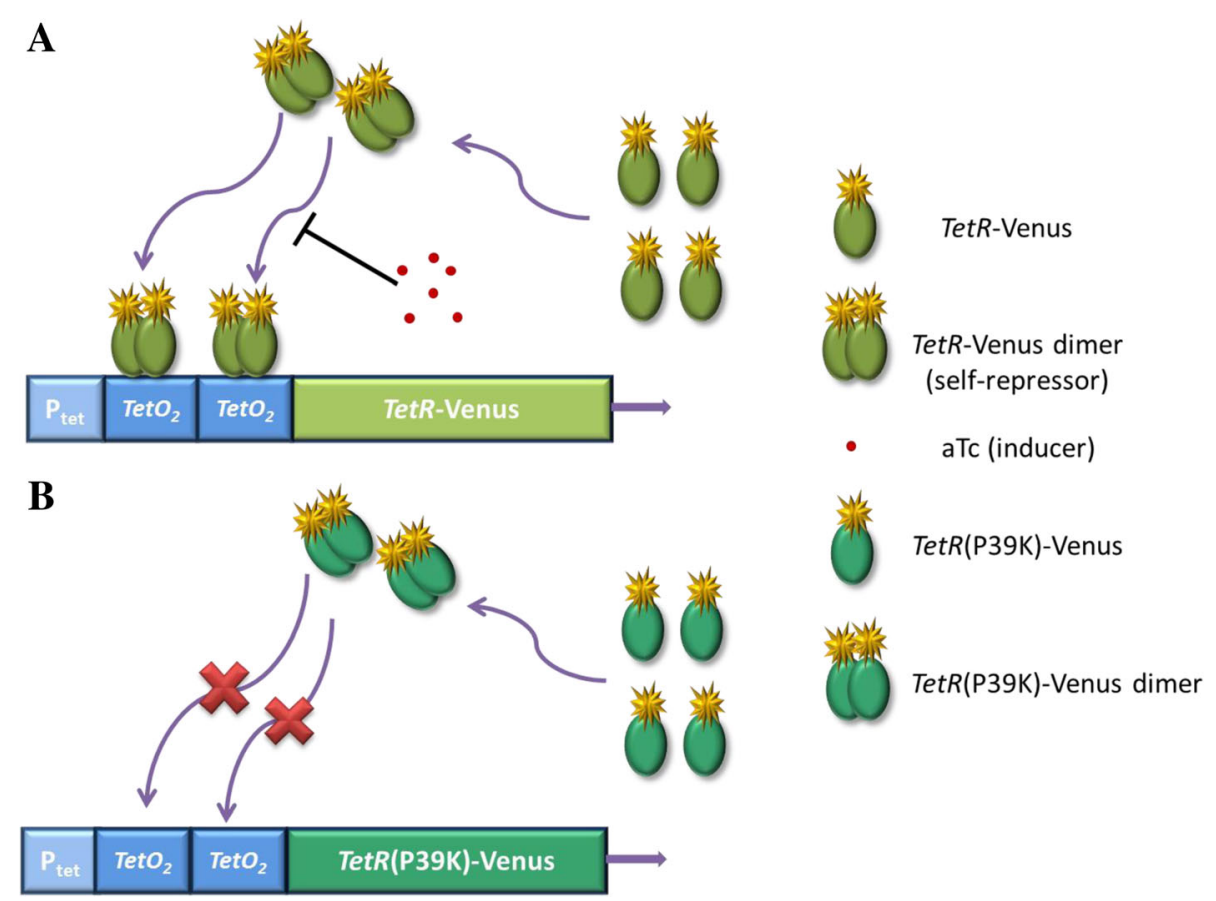

Fig. 1 Schematic illustrations of the self-repressing gene circuit (MG::PR-8T) and the non-self-repressing gene circuit (MG::PR-8T-P39K). a Two tet operator sequences $\left(\mathrm{TetO}_{2}\right)$ inserted downstream of the Ptet promoter are bound by TetR self-repressor dimers. In the absence of aTc (the inducers), TetR-Venus dimers bind to the operators. This interaction prevents the binding of RNA polymerase, thereby inhibiting the TetR-Venus fusion protein synthesis. When aTc diffuse into the cell, they bind to TetR, inducing an allosteric conformational change in the repressor protein which releases it from DNA, allowing for the possibility of the gene being switched into the "on" state. All of these constitute a self-repressing gene circuit. $\mathbf{b}$ The TetRP39K mutant is not capable of recognizing the operators and is unable to repress the TetR-Venus expression, constituting a non-self-repression gene circuit

given in Fig. 2a. It is important to note that cells grow normally (cell cycle 40-60 min) and have normal cell morphology even at high concentration of aTc (Fig. 4b, Additional file 1: Figure S8). Therefore, at this high aTc concentration, there seems no significant cytotoxicity on cells. In our control experiments, two state behaviors are not found in the strain of MG::PR-8 T-P39K with nonself-repressing gene circuit under the same conditions (Additional file 1: Figure S3, Additional file 1: Figure S7). This indicates that the two expression states of TetR were due to the self-repressing circuit, rather than other factors such as the influences of the inducers on the cell growth and ribosomal effects. Meanwhile, the other two strains of MG::PR-WT and MG::PR-1G even with selfrepressing gene circuit displayed only single peak distributions (Additional file 1: Figure S3). These results suggested that the two states appear only under certain specific conditions. This might explain why previous studies did not see bimodality on negative auto-regulation system.

\section{Fano factor and inhibition curve}

To further understand our experimental observations, we need to quantify the degrees of fluctuations. This can be measured by the Fano factor quantified as the variance of the observable (molecular number in the cell) divided by the mean value [35]. The Fano factor is equal to one $(F=1)$ if the distribution of the observable (the molecular number) is exactly Poisson. A large Fano factor implies significant statistical fluctuations deviating from Poisson. As the concentration of inducers increases, the Fano factor increases (Fig. 3a). When the concentration of inducer reached $1300 \mathrm{ng} /$ $\mathrm{mL}$, the change of Fano factor is more significant. Qualitatively, the Poisson distribution should be a good approximation for the individual "on" and "off" states when the observed distribution of fluorescence intensity is bimodal, because each gene state can produce proteins almost independently of gene switching. However, the overall Fano factor for the combined probability distribution of "on" and "off" states is much larger than the case of the zero or smaller inducer concentration. This is because the system is close to a two-peak (non-Poisson) distribution with different means summed together, producing large statistical fluctuations deviating from the single Poisson distribution. This indicates that two Poisson processes added together will not lead to a Poisson distribution. In this study, we measured the Fano 


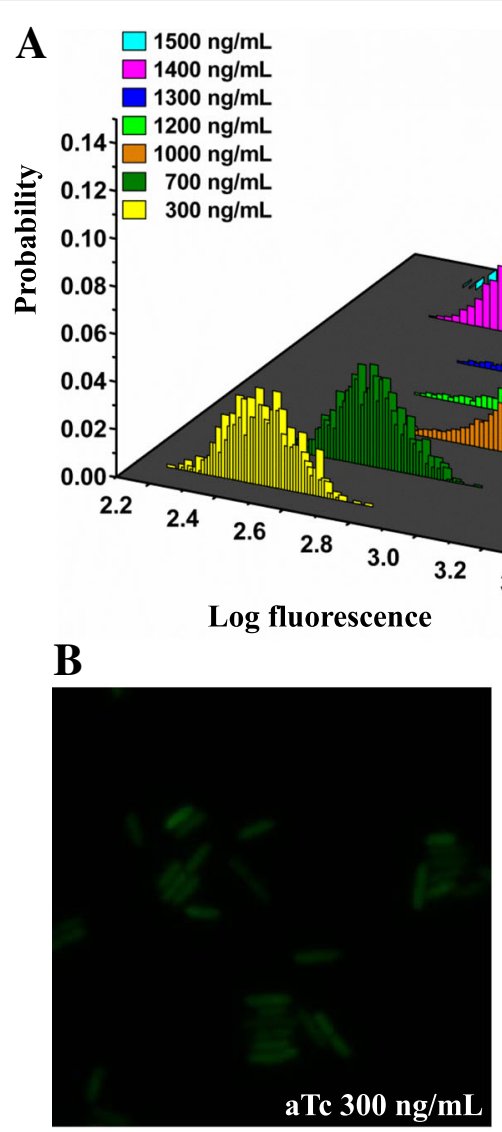

\section{$270 \min$}

D

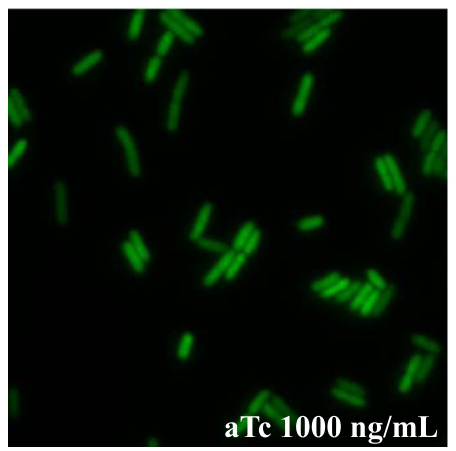

$\mathbf{E}$

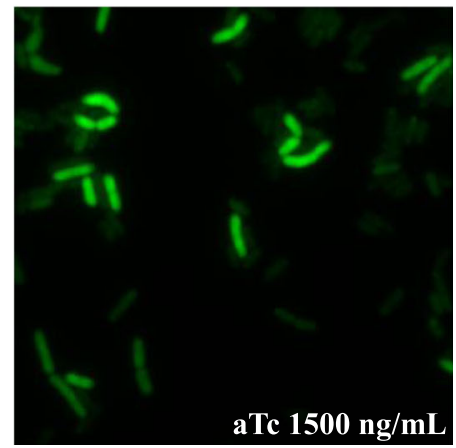

Fig. 2 Experimental expression distributions of the self-repressing gene circuit (MG.:PR-8 T) at different aTc concentrations observed under a microscope. a In M9 media with the inducer concentrations ranging from 300 to $1500 \mathrm{ng} / \mathrm{mL}$ of aTc, the resulting steady state fluorescence distributions show that the ratio of the populations of the bimodal fluorescence distributions depend on the aTc concentration. Seven color histograms represent different inducer concentrations. b-e Four representative fluorescence images at different concentrations of aTc (300, 700, 1000 , and $1500 \mathrm{ng} / \mathrm{mL}$ ) are selected

factor quantified as the variance of the fluorescence observable (proportional to the molecular number) divided by the mean value to quantify the relative degree of the variance or fluctuations. In other words, the phenotypic noise strength, defined as the quantity $\delta^{2} / \mu$, is a measure of the spread of expression levels in a population. Here we point out that even when the underlying population obeys the
Poisson distribution, the fluorescence observable which is proportional to the population size may not have a Fano factor equal to one. It is worth emphasizing here that we care more about the trend or the changes of the Fano factor upon inducer concentrations rather than the absolute values here. This can help us to understand the degree of the spread or fluctuations in protein expression distribution 

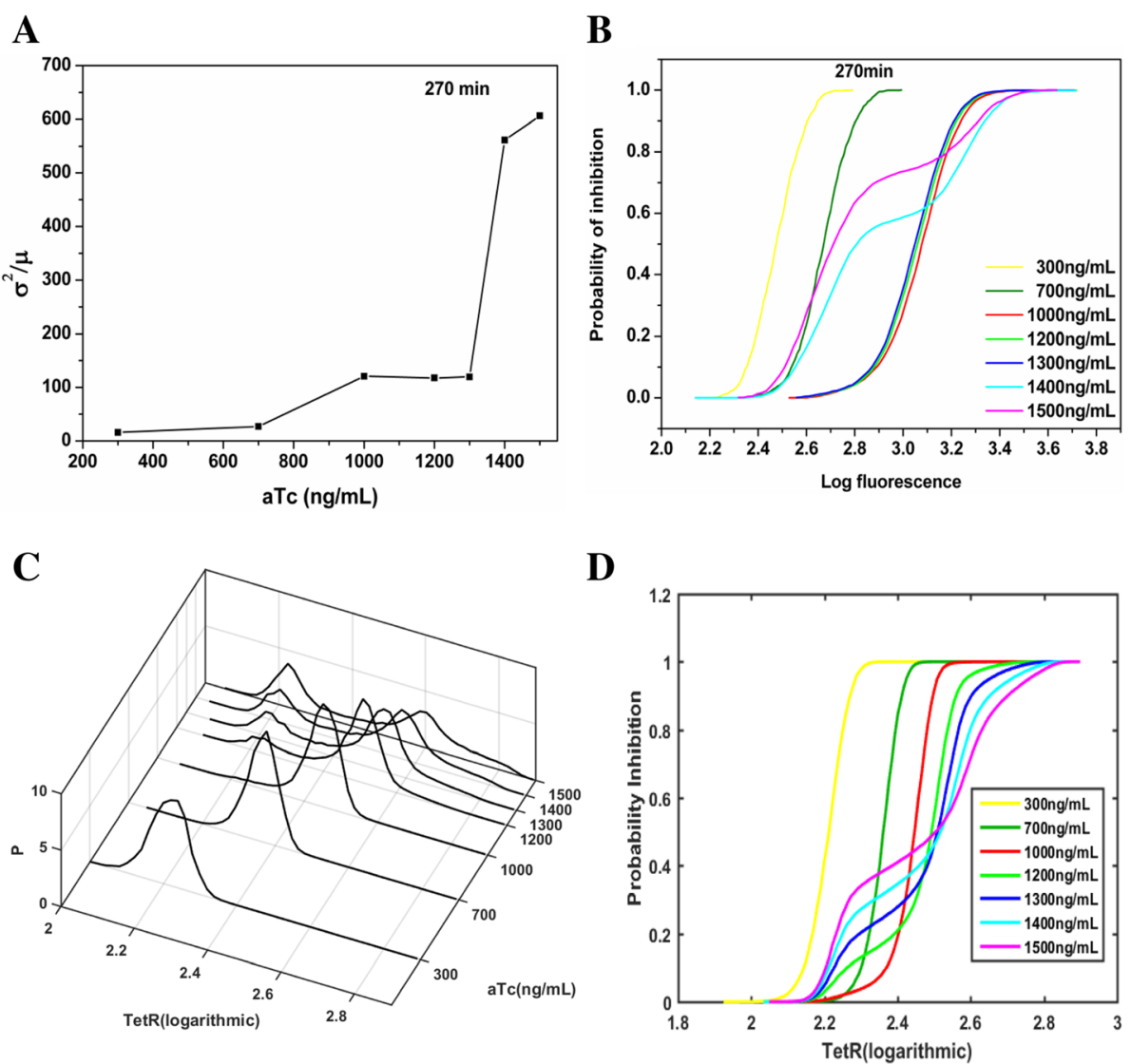

Fig. 3 The Fano factor curves and the probability of inhibition curves of the self-repressing gene circuit. a Dose-response of the Fano factor $\left(F=\sigma^{2} / \mu\right)$ of the TetR-Venus expression for the self-repressing gene circuit (MG::PR-8T) at different inducer concentrations. The Fano factor is defined as $\sigma^{2} / \mu$, where $\sigma^{2}$ and $\mu$ are the variance and the mean of the probability distribution. $\mathbf{b}$ The probability of inhibition curves of the MG::PR-8 T circuit at different inducer concentrations. Seven color histograms represent different inducer concentrations. The inhibition curves were obtained by the ratio of the cells with a fluorescence intensity lower than a certain value to the number of the total samples. c The probability distribution of the TetR proteins for the circuit of MG.:PR-8T with different concentrations of inducers from the stochastic simulation model. P( $n$ ) ( $z$ axis) represents the probability distribution of the TetR protein numbers ( $x$ axis), $n$ at different numbers of inducer (aTc) molecules ( $y$ axis). $\mathbf{d}$ The probability of inhibition curves of the model. The cumulative distribution functions of the simulation were obtained from Fig. 3c. Seven color histograms represent different inducer concentrations

upon inducer concentrations. The analysis of the coefficient of variation (CV) in Additional file 1: Figure S6 also illustrates this same conclusion.

Furthermore, we investigated the inhibition curve, which describes the proportion of the bacteria with a fluorescence intensity lower than a certain value (Fig. 3b). We can see that the proportion of the gene in its inhibited state first decreases at low concentrations of inducer (up to aTc concentration at $1200 \mathrm{ng} / \mathrm{mL}$ ) and then increases as the inducer concentration becomes higher. More inducers introduce more interactions with the TetR molecules. This leads to a reduction in the number of free TetR (Additional file 1: Figure S15), and also slows down the effective binding and unbinding of the TetR to the DNA (Details in Additional file 1: SI on page 10). The reduction of the binding rate will lead to the decrease of inhibition capability, and the decrease of unbinding rate will lead to the increase of inhibition capability. When the concentration of inducer aTc is low (higher free $T e t R$ concentration), the binding rate has a greater influence (dimer with square dependence on the free TetR concentration) on the overall inhibition capability than the unbinding rate (linear dependence on the free TetR concentration [43-52]). Therefore, at low inducer concentrations, when the inducer concentration increases (up to aTc concentration at $1200 \mathrm{ng} / \mathrm{mL}$ ), the free TetR concentration decreases. This leads to the reduction of the binding rate. Since binding has square dependence on the free TetR concentration and unbinding has linear dependence on the free TetR concentration, the binding has more significant influence than unbinding on inhibition capability at relatively low aTc concentration, i.e., relatively high free TetR concentrations. Therefore, the reduction of the binding leads to 
the decrease of the inhibition probability at this inducer concentration range (up to aTc concentration at $1200 \mathrm{ng} / \mathrm{mL}$ ).

At certain concentrations of inducer aTc (1200 ng/ $\mathrm{mL}$ ), the binding rate and unbinding rate have the same effect on the inhibition capability. If the concentration of the inducer further increases (at this concentration range of aTc $1200-1500 \mathrm{ng} / \mathrm{mL}$ ), the unbinding rate will have a greater influence on the inhibition capability, that is, the inhibition capability will increase as the inducer concentration increases. This is because unbinding has linear dependence on the free $T e t R$ concentration and binding has square dependence on the free TetR concentration; the unbinding has more significant influence than binding on inhibition capability at relatively high inducer concentration, i.e., at relatively low free TetR concentrations. At extremely high aTc concentrations (beyond $1900 \mathrm{ng} / \mathrm{mL}$ ), the toxicity from aTc as antibacterial agent to the cells becomes effective. It is therefore not feasible to observe the healthy cell expression distribution at this extremely high concentration of aTc.

\section{The dynamics of TetR expression in real time}

We have seen that the self-repressing circuit can give a bimodal distribution. In order to further explore the underlying mechanism of this behavior, we monitored the dynamics of TetR expression in real time. We tracked cells during their growth and division on a microscope with a FCS2 (Focht Chamber System 2, Bioptechs) system which provides aTc continuously to guarantee the cells growing in the right environments (continuous flow of adequate nutrients from fresh medium (M9) through the cells on agarose pad) and avoids potential issue of heterogeneity of the environments. As shown in Fig. 4b, upon aTc induction, two types of cell responses were observed: the fluorescence intensity either changed significantly or almost remained the same. When we track cells in real time, we can see that, some cells switch between bright and dim, while other cells stay with similar brightness (Fig. 4b). The resulting fluorescence distribution is thus bimodal and a fluorescence threshold can be defined for each cell in its most probable induction state. The use of a microfluidic device, coupled with cell tracking and fluorescence measurements, allows us to generate fluorescence trajectories for a single cell on reasonable time scales $(\sim 300 \mathrm{~min})$ for a single trajectory. Based on this, we collected 28 micro-colony movies and chose 163 fluorescence trajectories. We observed that the trajectories of a single-cell fluorescence fluctuated significantly. We collected about 8200 fluorescence intensity data points corresponding to the selected trajectories. Several representative trajectories with significant fluctuations were shown to demonstrate the existence of two states (From
Fig. 4a, b, Additional file 1: Figure S8, Additional file 2: Movie S1, Additional file 3: Movie S2 and Additional file 4: Movie S3).

\section{Two cell state identifications by hidden Markov chain modeling}

In order to explore the underlying mechanism of the bimodality, we collected the statistics of the fluorescence intensity obtained from the trajectories. As expected, the distribution of these intensities exhibits two peaks, which is similar to the results in Fig. 2a. Here, it is important to note that, due to the different growth conditions of the cells between the steady state and time-lapse experiments, there should be certain differences on the expression distribution curve. However, the images taken under both steady state and time-lapse experiments show two group of cells (bright and dim cells) (Fig. 2e and Fig. 4b). This indicates that the two peaks should come from the two groups of the cells on both experiments. Therefore, we believe that the two state populations uncovered from the single-cell trajectories are the same two state populations uncovered in the steady state experiments. The emergence of the two peaks suggests that most of the initial cells are either in a high expression state or in a low expression state in their progeny. We then used a Hidden Markov Chain Model (HMM) [36] to fit the real-time trajectories and identify the cell states and then simulate the distribution of the fluorescence intensity (Fig. 4c). To assign protein expression states and the rates of inter-conversion between them, we performed data fitting using the HMM. From the HMM analysis, we obtained a correlation coefficient of 0.975 between the measured and simulated trajectories after identifying the cell states and quantifying their switching rates. The simulated distribution fits with the measured distribution well. From the HMM analysis, we further determined the center positions of the peaks to be at 2.690 and at 2.933 in logarithm of fluorescence intensity. The variances of the individual peak distributions are at 0.085 and at 0.080 , respectively. For our system, the probability in the high expression state is around 0.401 , and we can also see that the probability in the low expression state is around 0.599 .

In the high expression state, the system will continue its behavior with a probability of 0.963 (the switching or residence time will be discussed in the next section). There is a small chance, with the probability of 0.037 , to switch to the low expression state from the high expression state. Meanwhile, there is additionally a probability of 0.023 that the system will switch to the high expression state from the low expression state, instead of remaining in the low expression state. 
A

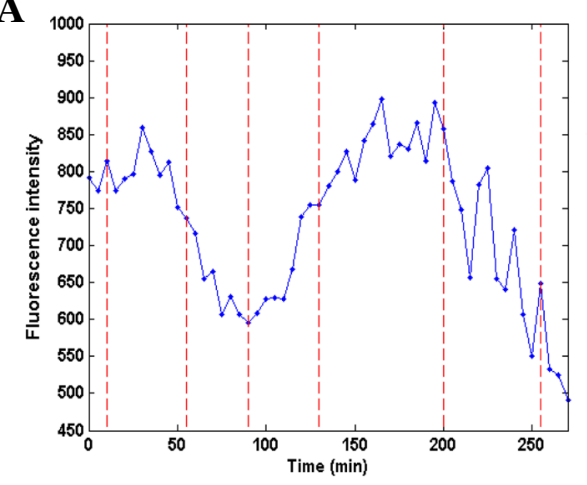

B
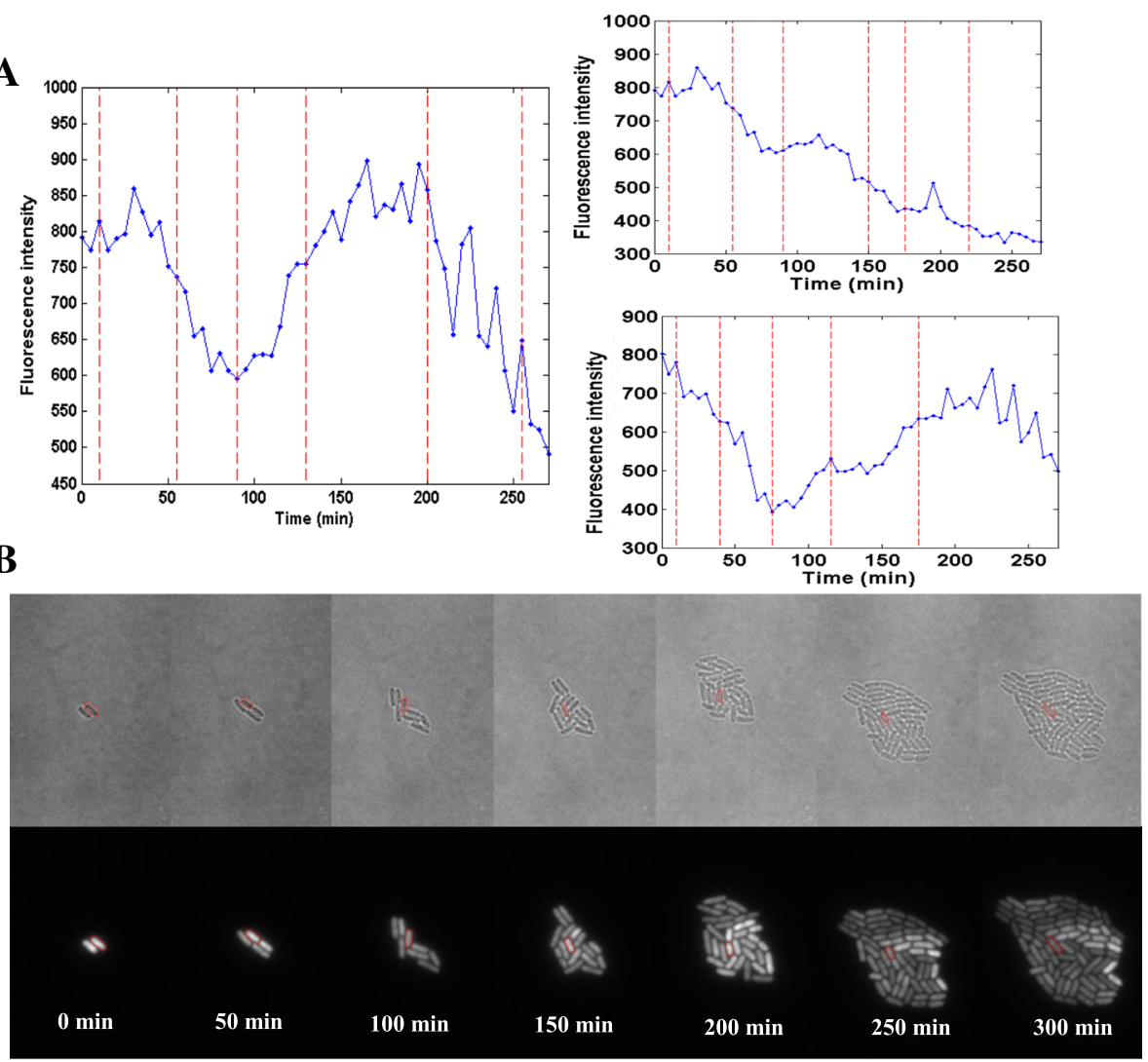

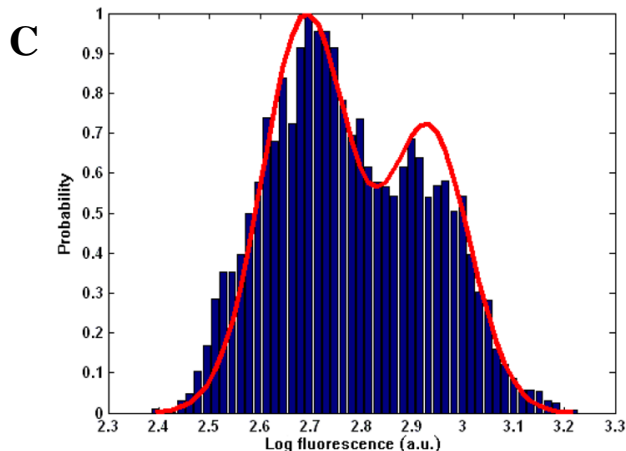

Fig. 4 The mean fluorescence intensity distribution of the dynamical trajectories for MG::PR-8T. Single-cell mean fluorescence intensities were captured every $5 \mathrm{~min}$. 28 micro-colonies were tracked by time-lapse microscopy. a Three representative single cell fluorescence trajectories induced by $1500 \mathrm{ng} / \mathrm{mL}$ aTc. Points represent experimental fluorescence values. Red vertical dashed lines demarcate cell divisions. $\mathbf{b}$ The bright field and fluorescent field images of the corresponding measurements in the time-lapse experiment. The cells corresponding to the fluorescence trajectory in Fig. 4 a are marked with red circles. The average of bacteria mean fluorescence intensity is 556, and the average cell cycle time is 46 min in this micro-colony. c The histogram gives the intensity distribution of the 163 single-cell fluorescence trajectories induced at $1500 \mathrm{ng} / \mathrm{mL}$ aTc collected from the time-lapse experiments. The red solid curve is the fitted intensity distribution from HMM

The average residence times of the protein expression states

To estimate the average residence times of the protein expression state, we distinguished the states from the trajectories using HMM analysis and calculated the residence times of each state (Additional file 1: Figure S11). For each trajectory, we counted the total residence times and the number of the state changes. The average residence times were calculated as the quotient of the total residence times and the number of states changed.

The length of the test fluorescence trajectory is finite and limited. This may lead to some errors in estimating the transition times. We take this into account in determining the time scale of the transitions (SI P8-P9). The average residence time of the high expression state is estimated to be about $92 \sim 103 \mathrm{~min}$, and that of the low 
expression state is estimated to be about 151 182 min. The average residence time can be used to quantify the switching time between two cell fates. Therefore, the switching time from high (low) expression to low (high) expression can be estimated to be about 92 103 (151 182) min. Through fluctuations, the bimodal distribution can be maintained in a dynamic balance between the high expression "on" state and the low expression "off" state. When the inducer concentration is fixed, the increasing number of proteins will promote the inhibition probability of gene switching. Therefore, the cells in the high expression state will have a tendency to migrate to the low expression state. Conversely, the cells with low expressions will be more likely to move towards the "on" state. Therefore, the cells in the low expression state will also have a tendency to migrate to the high expression state.

\section{Physical origin of the two cell fates}

Intuitively, from a molecular perspective, we know that the transcription process is suppressed when the promoter site of the DNA is occupied by a repressor (the gene is "off") and enhanced when the repressor is dissociated from DNA (the gene is "on").

According theoretical studies [32], when the binding/ unbinding is much faster relative to the synthesis/degradation, the gene regulatory network is under adiabatic limit. In this case, the "on" and "off" of gene states switch rapidly, but the protein molecules synthesize or degrade much slower in this self-repressor circuit. Therefore, the expression of the protein and associated gene are strongly coupled together. As soon as the proteins are synthesized, they will immediately bind to the gene. Therefore, the protein is always repressed (self-repressor) and the protein expression displays a single peak at the long time scale. When the binding/unbinding is lower or comparable relative to the synthesis/degradation, the gene regulatory network is in non-adiabatic limit. In this case, the gene state changes slowly. After the proteins are synthesized, they will take certain amount of time for binding. During this period of time, the gene state can be on without binding while after this time the gene will be off due to the protein binding to the gene. Therefore, there is a fraction of time the gene is in on and off state. This leads to the emergence of the protein distribution with two distinct peaks showing two different gene states.

Although we did not directly measure the binding/unbinding rate of TetR, recent single-molecule experiments [43-52], using force spectroscopy, fluorescence microscopy, and analog computation have determined the unbinding rate of some proteins bound to the DNA. The results showed that the unbinding rate depends on the concentration of freely diffusing proteins in the solution.
For the unbinding mechanism, Paramanathan et al. proposed a universal rapid rebinding model to quantitatively explain the kinetic process of competitor-induced dissociation. The protein bound to DNA undergoes rapid dissociation and rebinding in the vicinity of the inhibition site. Other proteins in this region can interact with the inhibition site to accelerate the unbinding rate. We suggest that this model can explain our experimental observed behaviors well. For the binding reaction, Tet $R$ dimer binds to DNA inhibition site to repress the gene expression, two TetR monomers combine to form a Tet $R$ dimer, and then the binding reaction can be simplified to a reaction between the gene and two TetR monomers. This leads to the quadratic dependence of the binding rate on the TetR concentrations. For the unbinding reaction, according to the rebinding model, the gene will react with the competitor. Due to the presence of the inducer, the conformation change of TetR will lead the Tet $R$ dimer to convert into monomers [53], so the competitor can be chosen as TetR monomer. This leads to the linear dependence of the unbinding rate with respect to the free TetR concentrations.

For dimer binding, the binding rate is expected to be proportional to the square of the concentration of the free TetR. The binding of aTc and TetR will lead to a decrease in the number of free TetR (Additional file 1: Figure S15). This will further lead to a decrease in the binding and unbinding rate between the TetR and the promoter. Therefore, when the inducer concentration is low, increasing the inducer concentration will decrease the free TetR and slow down the binding and unbinding rate of free TetR to the promoter. This will increase the probability of the gene being at the "on state" and "off state." When the free TetR concentration is not too low, the effect of binding (quadratic dependence on the free $T e t R$ concentration) is more significant compared to the effect of unbinding (linear dependence on free TetR concentration). As the aTc increases, free $T e t R$ decreases. This reduces the binding rate more significantly than the unbinding rate. The less effective binding is expected to lead to more chances of genes being at on state. Furthermore, the binding and unbinding rates are usually faster than the degradation rate of TetR, the resulting synthesis is faster. As a result of all above, the higher expressions emerge. This explains the shift of the expression peak from low to high as inducer concentration increases.

When the inducer concentration further increases to sufficiently high values, the free TetR molecules will be fewer, the binding and unbinding rate will also be lower. This will increase the residence time of genes being at "on state" and "off state." At the "on state," more TetR will be generated, and then bind with the inducer, corresponding to the high expression peak. However, when the free TetR concentration is very low, the effect of 
unbinding (linear dependence on the free TetR concentration) is more significant compared to the effect of binding (quadratic dependence on free TetR concentration). As the aTc increases further, free TetR decreases. This reduces the unbinding rate more significantly than the binding rate. The less effective unbinding is expected to lead to more chances of genes being at off state. As a result, the lower expressions emerge. This explains the shift of the expression peak from high to low as inducer concentration further increases.

When the average residence time of "off state" is longer than the average degradation time of TetR, the free TetR and the TetR binding with aTc will be degraded significantly. The resulting synthesis and degradation of the proteins can reach the steady state. This will make the low expression peak more stable. Further increases of the inducer concentrations will lead to less free Tet $R$ and the longer residence time of "off state." This leads to greater weighting of low expression peak rather than high expression peak.

\section{Stochastic simulations of bimodality}

To verify our explanation about emergence of two states on our TetR self-repressor system discussed above, we further explored the stochastic dynamics of selfregulative feedback genes through a mathematical model, which can be used to explain and simulate the experimental observations (Fig. 3c). The mathematical model clarifies the underlying mechanism of how bimodality emerges. Under faster regulation binding, the selfrepressor is forced to stay in the repressed state. This is because once produced, the regulatory protein immediately binds to the gene and therefore represses protein production. In our study, slower binding of the regulatory protein to the gene is realized through the inducer binding to the regulatory protein, which effectively blocks the ability of the protein to bind to the promoter. Under slower regulatory binding, the self-repressor may function in two different ways: it may bind to the DNA for some time and repress protein production, or unbind from the DNA for some time, leading to increased protein production. This generates two cell phenotypes. Furthermore, due to the intrinsic statistical fluctuations of the number of proteins, there is a possibility of switching between the high expression and low expression state. We have observed such phenotypic switching in real-time experiments. The simulation results are consistent with the experimental observations for the emergence of the two peak distributions in Fig. 2a.

On the other hand, the trajectories in Fig. 4a and Additional file 1: Figure S8 showed comparable growth rates in high expression state and in low expression state. It is possible that high expression cells in our study have not reached the threshold for significant metabolic burden to slow down the growth. The inhibition curves of the different inducer concentrations in Fig. $3 \mathrm{~b}$ and the dynamic balance by intrinsic fluctuations also imply that the bimodality of the protein expression distribution is not due to cell growth. The simulation results of Fig. 3d are also consistent with the experimental finding for inhibition probability shown in the Fig. $3 \mathrm{~b}$.

\section{Biomodality in self-repressing circuits at different affinities}

We constructed three self-repressing circuits with different affinities to the TetR protein [38] (relative affinity of TetR between WT, $8 \mathrm{~T}$, and $1 \mathrm{G}$ is 100,79 , and 13 ). We chose MG::PR-8 $\mathrm{T}$ as the main circuit of this study for its stability and bimodal behavior since we did not observe good reproducible bimodal behavior on the other two circuits under our current experimental conditions. We hypothesize that the differences in affinities between the gene circuits and the $T e t R$ protein are the main cause of the differences in behaviors. It is reasonable to expect that the same behavior such as bimodal behavior for the systems with different affinities requires different experimental conditions.

Here, we can give a more detailed possible explanation from the simulations. In the simulation, we used rate parameter $\mathrm{f} / \mathrm{h}$ to quantify and clarify the important role of affinity in the self-repressor. Different affinities correspond to different equilibrium constants $\mathrm{f} / \mathrm{h}$ in the inverse fashion. We started the unbinding/binding ration $\mathrm{f} / \mathrm{h}$ corresponding to $8 \mathrm{~T}$ circuit. Since the relative affinity of $1 \mathrm{G}$ circuit is lower than $8 \mathrm{~T}$, to stimulate the affinity of $1 \mathrm{G}$ circuit, we increased the value of $\mathrm{f} / \mathrm{h}$, and the results were given in Additional file 1: Figure S16. Bistability can still be observed but at much higher aTc concentration. As mentioned before, the cells can hardly survive in such an environment. This may explain why we did not observe bimodal behavior in the strain of MG::PR-1G. On the other hand, we also stimulated the WT circuit with higher affinity. The results are shown when the unbinding/binding ratio of $\mathrm{f} / \mathrm{h}$ decreases, in Additional file 1: Figure S17. Although bimodal behavior in principle can be reached at lower aTc concentrations, it is very difficult to distinguish and clearly separate the two states due to the fluctuations, noises and errors at lower concentrations of proteins in the strains of MG::PR-WT. In this study, the behavior of MG::PR-8 $\mathrm{T}$ is sufficient to illustrate that the selfrepressing circuits can become bimodal. Therefore, we conclude that the bimodality can emerge for self-repressor systems under certain experimental conditions.

\section{Biomodality and trend of biomodality upon inducer impacts and their relationships to TetR concentration dependence of binding/unbinding regulations}

Another issue of concern is that whether TetR concentration dependence is necessary for the bistability to 
occur. Based on the simulation results, we can see that the distribution with the unbinding rate independent on the concentration can still be bimodal. In other words, when the concentration dependence for unbinding is removed, we can also reach the bimodal distribution, as shown in the Additional file 1: Figure S18. However, the trends and shapes of the expression distribution with respect to the inducer concentration are not consistent with the experimental observations. We can see that when the inducer concentrations are high enough (such as $1400 \mathrm{ng} / \mathrm{mL}$ and $1500 \mathrm{ng} / \mathrm{mL}$ ), the steady state expression distribution can become bimodal, with two states of low and high expression levels, respectively. Meanwhile, the percentage of the cells in the low expression state gradually increases with the increase of the inducer concentrations (Fig. 2a). When the concentration dependence of unbinding is removed, the percentage of the high expression state gradually increases with the increase of the inducer concentrations when the bimodality is emerged, which is not consistent with the experimental observation.

To illustrate this more specifically, let us consider the following scenario. When the concentration of inducer is low, the concentration of free TetR is high, and the gene is mostly in the off state. Then, the gene expression would have a single peak distribution. Increasing the inducer concentration will decrease the free TetR concentration and slow down the binding rate of free TetR to the promoter, this will increase the probability of the gene being at the "on state." If we assume that the unbinding rate is constant, the probability of the gene being at the "off state" is invariant. Hence, the shift of gene expression peak is from low to high.

When the inducer concentration is further increased, the probability of "on state" would continue to increase. Due to the very fast expression rate, we will reach a higher expression peak corresponding to the higher probability of "on state." As the inducer aTc concentration increases further, the free Tet $R$ decreases and the probability of "on state" continues to increase, this leads to greater increase of the weight of high expression peak rather than low expression peak (Additional file 1: Figure S18). In this condition, the simulation results with unbinding rate independent of TetR concentration are not consistent with the experimental observations.

\section{Discussion}

Our study shows explicitly in this concrete gene circuit that different cell fates can emerge not only from the changes in the genes (such as mutations) but also from the changes in regulatory wirings or links through microenvironments without altering the gene itself. In fact, even when the topology of the wiring for the underlying gene regulatory network is fixed, there is still a possibility of cell phenotypic changes due to the changes in the regulation strengths induced by the environment. Furthermore, we observed in both real-time experiments and simulations that the cell phenotypes or fates can be switched from one to the other. We also obtained the average time of this switching which quantifies how difficult it is to communicate globally from one cell fate to the other. Therefore, using real-time trajectories, we determine both the speed and the underlying processes of the cell fate decision-making/phenotypic state-switching.

Epigenetic effects are often challenging to study in eukaryotic cells. Our study in bacteria illustrates how the environments can influence the cell fates and cell fate decision-making in a controllable way. The experiments in bacteria are relatively easy and straightforward to perform and control. The epigenetic and microenvironmental effects can be mimicked through the modulation of inducers in our study. The essence of the effects of the epigenetics is the change of the effective time scales of the regulatory binding/unbinding. Here the introduction of the inducers into the culture medium changes the microenvironment of the cell and leads to the changes of the time scales of the regulatory binding/unbinding, mimicking the effects of the epigenetics. This is an advantage of our approach. We plan to apply our method to a variety of core regulatory motifs and modules in the gene networks to investigate how the microenvironments or epigenetics influence the cell fates and the cell fate decision-making processes.

\section{Conclusions}

From the results of this experimental study, we suggest that different phenotypes can emerge from the same genotype under environmental changes, in this case that introduction of an inducer to a self-repressor circuit can, in certain conditions, lead to the emergence of two cell fates rather than the expected one fate. We observe the switching dynamics for cell fate decision-making between these two types of populations and propose a model to show that slow binding/unbinding, rather than stochastic fluctuations, may underlie the emergence of bimodality.

\section{Materials and methods}

\section{Strains and plasmid construction}

The tetracycline inducible promoter (PLtetO-1) and a strong ribosome binding site (RBS, BBa_B0030) were obtained from the plasmid of pZE11, which was kindly provided by Bujard [26]. The PLtetO-1 promoter has two $\mathrm{TetO} 2$ operator sites. This promoter is tightly repressed by the Tn10-encoded Tet repressor (TetR) and can be activated by a supply of anhydrotetracycline (aTc).

All strains were constructed using standard cloning techniques. The sequences of the PCR primers used in this study are shown in Additional file 1: Table S1. The 
fusion PCR was used to construct the TetR-Venus fusion protein. The protocol is based on two rounds of PCR: The first PCR uses TetR-F/Linker-R and Linker-F/ Venus- $R$ as primer pairs, whose templates are the plasmid pBR322 (Invitrogen ${ }^{\mathrm{rm}}$ ) and E. coli SX4 genomic DNA from Xie [23], respectively. In the second PCR, the fragments from the first round were amplified and fused using primers $T e t R-F / V e n u s-R$, resulting in the TetRVenus fragment. The TetR-Venus fusion protein was connected with the flexible linker, which provided the proper distance between $T e t R$ and Venus to avoid interference.

The entire TetR-Venus fragment was digested with $K p n \mathrm{I}$ and $X b a \mathrm{I}$ and ligated into the pZE11 vector backbone, which placed the TetR-Venus under the control of the PLtetO-1 promoter, yielding the plasmid pZE11PLtetO-1-TetR-Venus.

\section{Recombination of chromosomes}

E. coli K-12 MG1655 was chosen as the host strain of the self-repressing circuit model system due to its hypotoxicity and its known genomic sequence. It demonstrates good performance for the foreign protein expression and has been used in genetic studies in laboratories worldwide. This is crucial for the expressions of the regulatory proteins in the self-repressing circuit.

The CRIM (conditional-replication, integration, and modular) plasmid pAH150, the corresponding helper plasmids pINT-ts, and E. coli MG1655 (F- $\lambda$-ilvG-rfb50 rph-1), were kindly provided by Wanner [37]. The vectors are designed to integrate into the bacterial chromosome at different phage attachment sites. The PLtetO-1-TetR-Venus was inserted into the pAH150 at the $X h o I$ and $X b a I$ sites, and as-prepared plasmid pAH150-PLtetO-1-TetR-Venus (Additional file 1: Figure S1) was transformed into the DH5 $\lambda \lambda \operatorname{pir}^{+}$strain for amplification. The resultant plasmids were integrated into the chromosome of the MG 1655/pINT-ts strain by standard electro-transformation protocol. A single copy of the integrant was screened from the transformants: the self-repressing gene circuit MG1655::PLtetO-1-TetRVenus (MG::PR-WT).

\section{The construction of other self-repressing gene circuits}

We mutated the operators to change their affinities to the repressor (Additional file 1: Figure S2). The mutated PLtetO-1 gene circuits were amplified by PCR using pAH150-PLtetO-1-TetR-Venus as a template, Ptet-X-F $(X=8 \mathrm{~T}, 1 \mathrm{G})$ as a forward primer and Venus- $\mathrm{R}$ as a reverse primer. These were then inserted into the pAH150 plasmid [38]. The constructed plasmids were integrated into the chromosome of MG 1655 to obtain MG::PR-8 $\mathrm{T}$. We thus constructed a series of self-repressing circuits with different affinities to the TetR protein.

\section{Construction of the control non-regulatory gene circuit}

To investigate whether the bimodality phenomena is produced by the self-repressing circuit, we designed a nonregulatory gene circuit as a control (Fig. 1b). The TetR mutant was introduced by site-directed mutagenesis with the pAH150-PLtetO-1-8 T-TetR-Venus plasmid as a template and P39K-F and P39K-R as a forward and a reverse primer according to standard procedures. The purified product was integrated into MG1655 as mentioned above, generating the MG1655::PLtetO-1-8 T-TetR-P39KVenus (MG::PR-8 T-P39K) circuit. The differences in binding affinities of the mutated TetR (TetR-P39K) and the wild-type TetR (wtTetR) may be attributed to their different interactions with the Tet operator. The mutant amino acid decreased the interaction strengths between TetR-P39K and TetO, without affecting TetR and aTc interactions [39].

\section{Microscopy measurements and image analysis}

Cells were cultured overnight in M9 minimal media supplemented with vitamins (MEM vitamin solution, Gibco) containing appropriate antibiotics by shaking at $250 \mathrm{rpm}$ at $37^{\circ} \mathrm{C}$. For microscopy measurements, the overnight cultures were diluted 1000 -fold into $5 \mathrm{~mL}$ of $\mathrm{M} 9$ media with different aTc concentrations (300-1500 ng/mL) (Additional file 1: Table S2) and were continuously shaken at $250 \mathrm{rpm}$. After four and a half hours, the cultures were washed twice with sterile water by centrifugation. The resulting pellet was re-suspended in M9 media with appropriate antibiotics to an $\mathrm{OD}_{600}$ of $0.2-0.4$. Small gel pads were prepared using 3\% low-meltingtemperature agarose in M9 media between microscope slides. $0.4 \mu \mathrm{L}$ of the prepared cell cultures were dropped on each pad for imaging using a fluorescent microscope.

The fluorescence values of the single cells were measured using an inverted fluorescence microscope (Ti-E, Nikon) with automated stage and focus, equipped with a $\times 100$ oil immersion objective. We applied $514 \mathrm{~nm}$ of an argon ion laser and set the output power at $5 \mathrm{~mW}$ (only $10 \%$ of the laser beam into the microscope objective). For each experiment, images of about 3000 cells on each slide were collected using a cooled EM-CCD camera (iXon3EM DU-897, Andor, Connecticut, USA). These images were acquired by Metamorph. Data analysis was accomplished through a combination of manual and automated analysis using custom MATLAB code (Schnitzcells).

\section{The time-lapse experiment and image analysis}

Overnight cultures in M9 media at $37^{\circ} \mathrm{C}$ were diluted 1000 -fold into M9 media with $1500 \mathrm{ng} / \mathrm{mL}$ of aTc and agitated at $37^{\circ} \mathrm{C}$ for $3 \mathrm{~h}$. According to the preliminary experiment, the concentration of aTc in the time-lapse experiment was set as $1500 \mathrm{ng} / \mathrm{mL} .0 .4 \mu \mathrm{L}$ of washed and re-suspended cell cultures in M9 media were dropped 
onto an agarose pad (3\% agarose in M9 media with $1500 \mathrm{ng} / \mathrm{mL}$ of aTc). The agarose pad was covered with a cover slide, resulting in cell placement into a FCS2 chamber [40]. Bioptechs-FCS2 system settings were applied: we used a media flow rate of $1-2 \mu \mathrm{L} / \mathrm{s}$, a media temperature of $37^{\circ} \mathrm{C}$, and a microscope objective temperature of $35^{\circ} \mathrm{C}$. All settings were adapted for optimizing the $E$. coli cell monolayer growth without physically disturbing the cells. The laser can be tuned at 514 $\mathrm{nm}$ for the Venus protein, and the output laser power was set at $3 \mathrm{~mW}$ (only 10\% of the laser beam up into the microscope objective). The fluorescent images were periodically captured and recorded in the transmitted and fluorescent channels every 5 min over a period of around 6-7 h with an exposure time of $50 \mathrm{~ms}$. All images were acquired using both bright field imaging and fluorescent field imaging.

Based on the custom analysis codes (Schnitzcells) compiled by MATLAB from Elowitz's lab [41, 42], we applied some modifications to adapt to our requirements. Such improved analysis codes were used to identify and follow the cells, to reconstruct their lineage in time-lapse analysis by fluorescence imaging, and to identify cells in statistical analysis. Finally, we collected the fluorescent data of each cell at each time point for the following discussion.

\section{A simple mathematical model explaining the self- repressor bimodal distribution}

We simulated the stochastic dynamical process of the self-repression gene circuit under intrinsic fluctuations from the finite number of molecules in the cell. The dimer consists of two regulator proteins TetR where each can be bound on to the DNA promoter with rate $1 / 2 \mathrm{~h} n(\mathrm{n}-1)$. Meanwhile, the dimer can be dissociated from the promoter with rates $f * n$, and this rate is the unbinding rate which is proportional to the number $(n)$ of free TetR. [50]. The binding and unbinding reactions of TetR with DNA are listed as follows:

$$
\begin{aligned}
& A^{11}+2 \operatorname{Tet} \stackrel{\stackrel{h}{\rightarrow}}{\rightarrow} A^{10}, A^{10}+\operatorname{Tet} R \stackrel{f}{\rightarrow} A^{11}+2 \text { TetR }+ \text { Tet } R \\
& A^{11}+2 \operatorname{Tet} \stackrel{\stackrel{h}{\rightarrow}}{\rightarrow} A^{01}, A^{01}+\operatorname{Tet} R \stackrel{f}{\rightarrow} A^{11}+2 \operatorname{Tet} R+\operatorname{Tet} R \\
& A^{10}+2 \operatorname{Tet} \stackrel{\stackrel{h}{\rightarrow}}{\rightarrow} A^{00}, A^{00}+\operatorname{Tet} R \stackrel{f}{\rightarrow} A^{10}+2 \text { TetR }+ \text { TetR } \\
& A^{01}+2 \operatorname{Tet} \stackrel{\stackrel{h}{\rightarrow}}{\rightarrow} A^{00}, A^{00}+\operatorname{Tet} R \stackrel{f}{\rightarrow} A^{01}+2 \operatorname{Tet} R+\operatorname{Tet} R
\end{aligned}
$$

where $A^{\mathrm{ij}}$ denotes the unbound (bound) state of the gene that synthesize the Tet $R$ protein ( $i$ represents one regulator protein of the dimer binding and $j$ represents another regulator protein of the dimer binding, 1 and 0 represent on and off state of the genes, respectively). For the self-repression gene circuit, $A^{11}$ denotes that the gene is completely switched "on" when the operator of the gene is unoccupied and active. $A^{00}$ denotes that the gene is completely switched "off" when the operator of the gene is occupied and repressed. For the binding reaction, TetR dimer binds to DNA inhibition site to repress the gene expression, two TetR monomers bind to form a TetR dimer, and then the binding reaction can be simplified into a reaction between the gene and two $T e t R$ monomers. This leads to the quadratic dependence of the binding rate on the TetR concentrations. For the unbinding reaction, according to the rebinding model, the gene can react with the competitor. Due to the presence of the inducer, the conformation change of TetR can lead to the monomerization of TetR dimer [53], so the TetR monomer can act as competitor. This leads to the linear dependence of the unbinding rate with respect to the free TetR concentrations.

We combined the transcription and translation steps for simplicity. Then, the transcription-translation step is described as follows:

$$
\varnothing \stackrel{g_{i i j}}{\rightarrow} \operatorname{Tet} R \text { for } A^{i j}, \quad \operatorname{Tet} R \stackrel{k_{1}}{\rightarrow} \varnothing
$$

where $\varnothing$ is used to represent a protein sink or source. The source for synthesis comes from the gene activations while the sink comes from the protein degradations. $g_{\mathrm{ij}}$ is the protein synthesis rate when promoters are active or inactive for gene state $A^{\mathrm{ij}}$, respectively. When we added the inducers into the cells, the inducers aTc were found to bind with the regulator protein TetR. The resulting binding complex cannot bind effectively to the promoter and therefore cannot have a significant influence on regulating gene transcription for synthesizing the protein. The binding complex of aTc and TetR may also degrade. This process can be described as follows:

$$
\begin{aligned}
& \text { TetR }+I_{a T c} \underset{a}{\stackrel{b}{\rightleftarrows}} T a, \quad T a \stackrel{k_{2}}{\rightarrow} \varnothing \\
& E_{a T c} \underset{c}{\stackrel{c}{\rightleftarrows}} I_{a T c}
\end{aligned}
$$

where $T a$ denotes the binding complex of TetR and aTc. These binding complexes of aTc and TetR cannot occupy the binding site of the promoter. $I_{\mathrm{aTc}}$ and $E_{\mathrm{aTc}}$ denote the internal and the external inducer (in and out of the cells). The internal and external inducers can mutually diffuse through the cell membrane with diffusion coefficient $c$. We define $\omega=\mathrm{f} / \mathrm{k}$, which quantifies the ratio between the unbinding rate of TetR to the promoter and the speed of the Tet $R$ protein degradation. Likewise, the equilibrium constant $X \mathrm{eq}=\mathrm{f} / \mathrm{h}$ quantifies the relative balance between dissociation and binding of TetR to the promoter (Additional file 1: Table S3) and is directly related to the affinity. 


\section{Additional files}

\section{Additional file 1: Supplementary Text. Table S1-S3. Figure S1-S18.} Table S1. PCR primers. Table S2. The corresponding relationship between the mass concentration and the molar concentration. Table S3. Rate constants in mathematical model. Figure S1. The recombinant plasmid map. Figure S2. Diagram of synthetic circuit constructs. Figure S3. The distributions of mean fluorescence intensity of MG::PR-WT and MG.:PR-1G. Figure S4. The distributions of mean fluorescence intensity of MG::PR-8 T. Figure S5. Total probability of the DNA in the bound state. Figure S6. Fano factor curves and the overall coefficient of variation (CV) curves. Figure S7. Expression distributions of MG.:PR-8 T-P39K. Figure S8. The representative trajectories for the MG::PR-8T strain. Figure S9. The fluorescence images of MG::PR-8 T-P39K. Figure S10. A representative trajectory collected at $50 \mathrm{ng} / \mathrm{mL}$

Figure S11. The schematic diagram of calculating the average residence time (each frame lasts $5 \mathrm{~min}$ ). Figure $\mathbf{S 1 2}$. The bifurcation diagram in total number of TetR (in $\mathrm{nmol} / \mathrm{L}$ ) molecules and aTc (in ng/ $\mathrm{mL}$ ) molecules. Figure S13. The bifurcation diagram in total number of TetR (in $\mathrm{nmol} / \mathrm{L}$ ) molecules and aTc (in $\mathrm{nmol} / \mathrm{L}$ ) molecules. Figure S14. The simulated steady state probability distribution in total TetR (in mol/ $\mathrm{L}$ ) and $\mathrm{aTc}$ (in $\mathrm{mol} / \mathrm{L}$ ) molecules. Figure S15. The simulated steady state probability distribution in free TetR (in mol/L) and aTc (in mol/L) molecules. Figure $\mathbf{S 1 6}$. The simulated steady state probability distribution with much higher aTc concentration. Figure S17. The simulated steady state probability distribution in different aTc concentrations. Figure S18. The simulated steady state probability distribution in different aTc concentrations when the unbinding rate is assumed not to be concentration dependent. (DOCX $4940 \mathrm{~kb}$ )

Additional file 2: Movie S1. This movie file shows a time-lapse microscopy of MG.:PR-8 T strains continuously induced with $1500 \mathrm{ng} / \mathrm{mL}$ of aTc at a constant temperature of $37^{\circ} \mathrm{C}$. The total time of the movie is 225 min with a rate of one image every $5 \mathrm{~min}$. (AVI $203 \mathrm{~kb}$ )

Additional file 3: Movie S2. This movie file shows a time-lapse microscopy of MG::PR-8 T strains continuously induced with $1500 \mathrm{ng} /$ $\mathrm{mL}$ of aTc at a constant temperature of $37^{\circ} \mathrm{C}$. The total time of the movie is $375 \mathrm{~min}$ with a rate of one image every $5 \mathrm{~min}$. (AVI $273 \mathrm{~kb}$ )

Additional file 4: Movie S3. This movie file shows a time-lapse microscopy of MG::PR-8 T strains continuously induced with $50 \mathrm{ng} / \mathrm{mL}$ of aTc at a constant temperature of $37^{\circ} \mathrm{C}$. The total time of the movie is $112 \mathrm{~min}$ with a rate of one image every $8 \mathrm{~min}$. (AVI $74 \mathrm{~kb}$ )

\section{Acknowledgments}

We thank H. Bujard and B.L. Wanner for providing PZE11 and CRIM vector systems, respectively, as well as for providing detailed information on their origins and growth conditions. We would also like to thank X. S. Xie for providing E. coli SX4

\section{Authors' contributions}

$\mathrm{ZLJ}$ and $\mathrm{LT}, \mathrm{XNF}, \mathrm{QZD}$, and JW contributed to the experimental design. ZL $L T, X N F$, and QZD conducted the experiments. $K Z, Q L, Z L J, L T, X F$, and JW contributed to the data interpretation. ZL, LT, XNF, KZ, QL, EKW, and JW contributed to writing and revising the manuscript. All authors read and approved the final manuscript.

\section{Funding}

This work was supported by the National Science Foundation (NSF) with grant number NSF-PHY76066 and NSF-CHE-1808474, the National Science Foundation of China (NSFC) with grant number 91430217, and the Ministry of Science and Technology (MOST) of China with grant number 2016YFA0203200.

\section{Availability of data and materials}

All data generated or analyzed during this study are included in this published article and its supplementary information files.

\section{Ethics approval and consent to participate}

Not applicable.

\section{Competing interests}

The authors declare that they have no competing interests.

\section{Author details}

${ }^{1}$ State Key Laboratory of Electroanalytical Chemistry, Changchun Institute of Applied Chemistry, Chinese Academy of Sciences, Changchun, Jilin 130022, China. ${ }^{2}$ Department of Chemistry, Physics and Applied Mathematics, State University of New York at Stony Brook, Stony Brook, New York 11794-3400, USA. ${ }^{3}$ College of Physics, Jilin University, Changchun, Jilin 130012, China.

Received: 28 December 2018 Accepted: 20 May 2019

Published online: 15 June 2019

\section{References}

1. Elowitz MB, Levine AJ, Siggia ED, Swain PS. Stochastic gene expression in a single cell. Science. 2002;297:1183-6.

2. Kaern M, Elston TC, Blake WJ, Collins JJ. Stochasticity in gene expression: from theories to phenotypes. Nat Rev Genet. 2005;6:451-64.

3. Becskei A, Kaufmann BB, van Oudenaarden A. Contributions of low molecule number and chromosomal positioning to stochastic gene expression. Nat Genet. 2005;37:937-44.

4. Swain PS, Elowitz MB, Siggia ED. Intrinsic and extrinsic contributions to stochasticity in gene expression. P Natl Acad Sci USA. 2002;99:12795-800.

5. Raj A, van Oudenaarden A. Nature, Nurture, or chance: stochastic gene expression and its consequences. Cell. 2008;135:216-26.

6. Qi H, Blanchard A, Lu T. Engineered genetic information processing circuits. WIREs Syst Biol Med. 2013;5:273-87.

7. Greenbury SF, Ahnert SE. The organization of biological sequences into constrained and unconstrained parts determines fundamental properties of genotype-phenotype maps. J R Soc Interface. 2015;12:20150724.

8. Fitzgerald O, Haroon M, Giles JT, Winchester R. Concepts of pathogenesis in psoriatic arthritis: genotype determines clinical phenotype. Arthritis Res The. 2015;17:1-11.

9. Yip L, Nikiforova MN, Yoo JY, Mccoy KL, Stang MT, Armstrong MJ, Nicholson KJ, Ohori NP, Coyne C, Hodak SP, Ferris RL, LeBeau SO, Nikiforov YE, Carty SE. Tumor genotype determines phenotype and disease-related outcomes in thyroid cancer: a study of 1510 patients. Ann Surg. 2015:262:519.

10. Katsonis $P$, Lichtarge $O$. A formal perturbation equation between genotype and phenotype determines the evolutionary action of protein-coding variations on fitness. Genome Res. 2014;24:2050-8.

11. Cheng Q, Yang W, Raimondi SC, Pui CH, Relling MV, Evans WE. Karyotypic abnormalities create discordance of germline genotype and cancer cell phenotypes. Nat Genet. 2005;37:878.

12. Wang J, Xu L, Wang EK. Potential landscape and flux framework of nonequilibrium networks: robustness, dissipation, and coherence of biochemical oscillations. PMC Biophys. 2008;105:12271-6.

13. Wang J, Zhang K, Xu L, Wang EK. Quantifying the Waddington landscape and biological paths for development and differentiation. P Natl Acad Sci USA. 2011;108:8257-62.

14. Wang J. Landscape and flux theory of non-equilibrium dynamical systems with application to biology. Adv Phys. 2015;64:1-137.

15. Sasai M, Wolynes PG. Stochastic gene expression as a many-body problem. P Natl Acad Sci USA. 2003;100:2374-9.

16. Balazsi G, van Oudenaarden A, Collins JJ. Cellular decision making and biological noise: from microbes to mammals. Cell. 2011;144:910-25.

17. Liao C, Lu T. A minimal transcriptional controlling network of regulatory $T$ cell development. J Phys Chem B. 2013;117:12995-3004.

18. Nevozhay D, Adams R, Murphy K, Josic K, Balázsi G. Negative autoregulation linearizes the dose response and suppresses the heterogeneity of gene expression. P Natl Acad Sci USA. 2009;106:5123-8.

19. Klumpp S, Zhang Z, Hwa T. Growth rate-dependent global effects on gene expression in bacteria. Cell. 2009;139(7):1366-75.

20. Liao C, Blanchard AE, Lu T. An integrative circuit-host modelling framework for predicting synthetic gene network behaviours. Nat Microbiol. 2017;2(12): 1658.

21. Blanchard AE, Liao C, Lu T. Circuit-host coupling induces multifaceted behavioral modulations of a gene switch. Biophys J. 2018;114(3):737.

22. Stricker J, Cookson S, Bennett MR, Mather WH, Tsimring LS, Hasty J. A fast, robust and tunable synthetic gene oscillator. Nature. 2008;456:516.

23. Yu J, Xiao J, Ren XJ, Lao KQ, Xie XS. Probing gene expression in live cells, one protein molecule at a time. Science. 2006;311:1600-3. 
24. Thattai $M$, van Oudenaarden A. Intrinsic noise in gene regulatory networks. P Natl Acad Sci USA. 2001;98:8614-9.

25. Becskei A, Serrano L. Engineering stability in gene networks by autoregulation. Nature. 2000;405:590-3.

26. Lutz $\mathrm{R}$, Bujard $\mathrm{H}$. Independent and tight regulation of transcriptional units in Escherichia coli via the LacR/O, the TetR/O and AraC/I-1-I-2 regulatory elements. Nucleic Acids Res. 1997;25:1203-10.

27. Hasty J, McMillen D, Collins JJ. Engineered gene circuits. Nature. 2002;420: 224-30.

28. Austin DW, Allen MS, McCollum JM, Dar RD, Wilgus JR, Sayler GS, Samatova NF, Cox CD, Simpson ML. Gene network shaping of inherent noise spectra. Nature. 2006;439:608-11.

29. Maithreye R, Sarkar RR, Parnaik VK, Sinha S. Delay-induced transient increase and heterogeneity in gene expression in negatively auto-regulated gene circuits. PLoS One. 2008;3:e2972.

30. Guet CC, Elowitz MB, Hsing WH, Leibler S. Combinatorial synthesis of genetic networks. Science. 2002;296:1466-70.

31. Kepler TB, Elston TC. Stochasticity in transcriptional regulation: origins, consequences, and mathematical representations. Biophys J. 2001;81: 3116-36.

32. Hornos JEM, Schultz D, Innocentini GCP, Wang J, Walczak AM, Onuchic JN, Wolynes PG. Self-regulating gene: an exact solution. Phys Rev E. 2005;72: 051907

33. Feng HD, Han B, Wang J. Adiabatic and non-adiabatic non-equilibrium stochastic dynamics of single regulating genes. J Phys Chem B. 2010;115: 1254-61.

34. Artyomov MN, Das J, Kardar M, Chakraborty A. Purely stochastic binary decisions in cell signaling models without underlying deterministic bistabilities. P Natl Acad Sci USA. 2007;104:18958.

35. Fano U. The fluctuations of the number of ions. Phys Rev. 1947;72:26-9.

36. Baum LE, Petrie T. Statistical inference for probabilistic functions of finite state Markov chains. Ann Math Stat. 1966;37:1554-63.

37. Haldimann A, Wanner BL. Conditional-replication, integration, excision, and retrieval plasmid-host systems for gene structure-function studies of bacteria. J Bacterial. 2001;183:6384-93.

38. Wissmann A, Meier I, Hillen W. Saturation mutagenesis of the Tn10-encoded Tet operator-01 - identification of base-pairs involved in Tet repressor recognition. J Mol Biol. 1998;202:397-406.

39. Helbl V, Hillen W. Stepwise selection of TetR variants recognizing tet operator 4C with high affinity and specificity. J Mol Biol. 1998;276:313-8.

40. Elf J, Li GW, Xie XS. Probing transcription factor dynamics at the singlemolecule level in a living cell. Science. 2007;316:1191-4.

41. Rosenfeld N, Young JW, Alon U, Swain PS, Elowitz MB. Gene regulation at the single-cell level. Science. 2005:307:1962-5.

42. Young JW, Locke JCW, Altinok A, Rosenfeld N, Bacarian T, Swain PS, Mjolsness E, Elowitz MB. Measuring single-cell gene expression dynamics in bacteria using fluorescence time-lapse microscopy. Nat Protoc. 2012;7:80-8.

43. Sing CE, Cruz MODL, Marko JF. Multiple-binding-site mechanism explains concentration-dependent unbinding rates of DNA-binding proteins. Nucleic Acids Res. 2014:42(6):3783-91.

44. Paramanathan $T$, Reeves $D$, Friedman $L$, et al. A general mechanism for competitor-induced dissociation of molecular complexes. Nat Commun. 2014;5:5207.

45. Cocco S, Marko JF, Monasson R. Stochastic ratchet mechanisms for replacement of proteins bound to DNA. Phys Rev Lett. 2014;112(23):238101.

46. Kunzelmann S, Morris C, Chavda AP, et al. Mechanism of interaction between single-stranded DNA binding protein and DNA. Biochemistry. 2010;49(5):843-52.

47. Gibb B, Ling FY, et al. Concentration-dependent exchange of replication protein a on single-stranded DNA revealed by single-molecule imaging. PLoS One. 2014;106(2):274a.

48. Graham JS, Johnson RC, Marko JF. Concentration-dependent exchange accelerates turnover of proteins bound to double-stranded DNA. Nucleic Acids Res. 2011;39(6):2249.

49. Chen TY, Cheng YS, Huang PS, et al. Facilitated unbinding via multivalencyenabled ternary complexes: new paradigm for protein-DNA interactions. Accounts Chem Res. 2018:51(4):860-8.

50. Chen TY, Santiago AG, Jung W, et al. Concentration- and chromosomeorganization-dependent regulator unbinding from DNA for transcription regulation in living cells. Nat Commun. 2015;6:7445.
51. Dahlke K, Sing CE. Facilitated dissociation kinetics of dimeric nucleoidassociated proteins follow a universal curve. Biophys J. 2017:112(3):543.

52. Hadizadeh N, Johnson RC, Marko JF. Facilitated dissociation of a nucleoid protein from the bacterial chromosome. J Bacteriol. 2016;198(12):1735.

53. Kedracka-Krok S, Wasylewski Z. Adifferential scanning calorimetry study of tetracycline repressor. Eur J Biochem. 2003;270:4564-73.

\section{Publisher's Note}

Springer Nature remains neutral with regard to jurisdictional claims in published maps and institutional affiliations.
Ready to submit your research? Choose BMC and benefit from:

- fast, convenient online submission

- thorough peer review by experienced researchers in your field

- rapid publication on acceptance

- support for research data, including large and complex data types

- gold Open Access which fosters wider collaboration and increased citations

- maximum visibility for your research: over $100 \mathrm{M}$ website views per year

At BMC, research is always in progress.

Learn more biomedcentral.com/submissions 